\title{
A FREDHOLM THEORY FOR A CLASS OF FIRST-ORDER ELLIPTIC PARTIAL DIFFERENTIAL OPERATORS IN $\boldsymbol{R}^{n}$
}

\author{
HOMER F. WALKER
}

\begin{abstract}
The objects of interest are linear first-order elliptic partial differential operators with domain $H_{1}\left(\boldsymbol{R}^{n} ; \boldsymbol{C}^{k}\right)$ in $L_{2}\left(\boldsymbol{R}^{n} ; \boldsymbol{C}^{k}\right)$, the first-order coefficients of which become constant and the zero-order coefficient of which vanishes outside a compact set in $\boldsymbol{R}^{n}$. It is shown that operators of this type are "practically" Fredholm in the following way: Such an operator has a finite index which is invariant under small perturbations, and its range can be characterized in terms of the range of an operator with constant coefficients and a finite index-related number of orthogonality conditions.
\end{abstract}

0 . Introduction. As usual, let $L_{2}\left(\boldsymbol{R}^{n} ; \boldsymbol{C}^{k}\right)$ denote the Hilbert space of equivalence classes of $\boldsymbol{C}^{k}$-valued functions on $\boldsymbol{R}^{n}$ whose absolute values are Lebesguesquare-integrable over $\boldsymbol{R}^{n}$. Let $H_{1}\left(\boldsymbol{R}^{n} ; \boldsymbol{C}^{k}\right)$ denote the Hilbert space consisting of those elements of $L_{2}\left(\boldsymbol{R}^{n} ; \boldsymbol{C}^{k}\right)$ which have (strong) first partial derivatives in $L_{2}\left(\boldsymbol{R}^{n} ; \boldsymbol{C}^{k}\right)$. Denote the usual norms on $L_{2}\left(\boldsymbol{R}^{n} ; \boldsymbol{C}^{k}\right)$ and $H_{1}\left(\boldsymbol{R}^{n} ; \boldsymbol{C}^{k}\right)$ by \|\| and \|\|$_{1}$, respectively. Consider a linear first-order partial differential operator

$$
A_{0} u(x)=\sum_{i=1}^{n} A_{i} \frac{\partial}{\partial x_{i}} u(x)
$$

with domain $H_{1}\left(\boldsymbol{R}^{n} ; C^{k}\right)$ which has constant coefficients and no zero-order term. Suppose that $A_{0}$ is elliptic in the sense that

$$
\operatorname{det}\left|\sum_{i=1}^{n} A_{i} \xi_{i}\right| \neq 0
$$

for all nonzero $\xi$ in $R^{n}$. Then, given a positive number $R$, let $E\left(A_{0}, R\right)$ denote the set of all linear first-order partial differential operators $A$ of the form

$$
A u(x)=\sum_{i=1}^{n} A_{i}(x) \frac{\partial}{\partial x_{i}} u(x)+B(x) u(x)
$$

with domain $H_{1}\left(R^{n} ; C^{k}\right)$, the $k \times k$ coefficient matrices of which are such that the following conditions are satisfied:

(i) The coefficients $A_{i}(x)$ and $B(x)$ are continuous complex-valued functions of $x$ on $\boldsymbol{R}^{n}$, and the coefficients $A_{i}(x)$ have continuous first derivatives.

Received by the editors March 26, 1971.

AMS 1969 subject classifications. Primary 3544, 3530, 4765, 3513.

Key words and phrases. First-order elliptic operators, indices of elliptic operators, Fredholm operators.

Copyright (C) 1972, American Mathematical Society 
(ii) The operator $A$ is elliptic, i.e. $\operatorname{det}\left|\sum_{i=1}^{n} A_{i}(x) \xi_{i}\right| \neq 0$ for all $x$ and all nonzero $\xi$ in $\boldsymbol{R}^{n}$.

(iii) The coefficients of $A$ are equal to the coefficients of $A_{0}$ outside the ball $B_{R}^{n}=\left\{x \in \boldsymbol{R}^{n}:|x| \leqq R\right\}$.

The operators in $E\left(A_{0}, R\right)$ are those elliptic operators obtained by adding a "perturbing" operator to $A_{0}$ whose coefficients vanish outside the ball $B_{R}^{n}$. The operator $A_{0}$ will be referred to as the unperturbed operator of the set $E\left(A_{0}, R\right)$.

The operators in $E\left(A_{0}, R\right)$ include operators of the type considered in the LaxPhillips theory of scattering for evolving systems whose evolution is governed by a symmetric hyperbolic system of partial differential equations [6]. In particular, the proper Hilbert space in which to construci this scattering theory is the orthogonal complement in $L_{2}\left(\boldsymbol{R}^{n}, \boldsymbol{C}^{k}\right)$ of the null-space of an operator which belongs to $E\left(A_{0}, R\right)$ for some unperturbed elliptic operator $A_{0}$ and some positive $R$. This fact provided the motivation for an earlier paper by the author [7] in which it is shown that the operators in $E\left(A_{0}, R\right)$ enjoy certain properties of Fredholm operators, namely, that the dimension of the null-space of an operator in $E\left(A_{0}, R\right)$ is finite and, in a certain sense, depends upper-semi-continuously on the operator. However, the range of an operator in $E\left(A_{0}, R\right)$ is never closed, and so such an operator cannot be Fredholm according to the usual definition. The objective of this paper is to show that, nevertheless, an operator in $E\left(A_{0}, R\right)$ is "practically" Fredholm in the following way: Such an operator has a finite index which is invariant under small perturbations, and its range can be determined in terms of the range of $A_{0}$ and a finite index-related number of orthogonality conditions.

1. Preliminary discussion and summary of results. Given a positive $R$ and an unperturbed elliptic operator $A_{0}$, let $A$ be an operator in $E\left(A_{0}, R\right)$. In the case of one independent variable, the equation $A u=0$ has no nontrivial solution which is square-integrable in absolute value over $\boldsymbol{R}^{\mathbf{1}}$; therefore, as in [7], it will be assumed that the number of independent variables is at least two. For an operator $A$ in $E\left(A_{0}, R\right)$, there exist positive constants $c_{1}$ and $c_{2}$ depending on $A$ such that the standard elliptic estimate

$$
\|u\|_{1} \leqq c_{1}\|u\|+c_{2}\|A u\|
$$

holds for all $u$ in $H_{1}\left(\boldsymbol{R}^{n} ; \boldsymbol{C}^{k}\right)$ [6]. From this estimate, it follows that $H_{1}\left(\boldsymbol{R}^{n} ; \boldsymbol{C}^{k}\right)$ is a natural domain for such an operator in the sense that the operator is closed on $H_{1}\left(\boldsymbol{R}^{n} ; \boldsymbol{C}^{k}\right)$ and its adjoint operator also has domain $H_{1}\left(\boldsymbol{R}^{n} ; \boldsymbol{C}^{k}\right)$. (For a discussion of the identity of the weak and strong extensions of first-order operators, see [2].) In fact, it is easily seen that $A_{0}^{*}$ is an unperturbed elliptic operator and that $A^{*}$ is an operator in $E\left(A_{0}^{*}, R\right)$. Consider the set

$$
M\left(A_{0}, R\right)=\left\{u \in H_{1}\left(R^{n} ; C^{k}\right): \text { support } A_{0} u \subseteq B_{R}^{n}\right\}
$$


and note that $M\left(A_{0}, R\right)$ contains the null-space $N(A)$ of every operator $A$ in $E\left(A_{0}, R\right)$. It is shown in [7] that every subset of $M\left(A_{0}, R\right)$ which is bounded in $H_{1}\left(\boldsymbol{R}^{n} ; \boldsymbol{C}^{k}\right)$ is relatively compact in $L_{2}\left(\boldsymbol{R}^{n} ; \boldsymbol{C}^{k}\right)$, and it follows that the dimension of $N(A)$ is finite for every $A$ in $E\left(A_{0}, R\right)$. Furthermore, for each $A$ in $E\left(A_{0}, R\right)$, there exists a positive constant $c$ for which the estimate

$$
\|u\| \leqq c\|A u\|
$$

holds for every $u$ in $M\left(A_{0}, R\right)$ which is orthogonal to $N(A)$. Note that if $A$ and $A^{\prime}$ are two operators in $E\left(A_{0}, R\right)$, then it follows from the boundedness of the coefficients of the operator $\left(A-A^{\prime}\right)$ that there exists a positive constant $c$ for which the estimate

$$
\left\|\left(A-A^{\prime}\right) u\right\| \leqq c\|u\|_{1}
$$

holds for all $u$ in $H_{1}\left(\boldsymbol{R}^{n} ; \boldsymbol{C}^{k}\right)$. The constant $c$ may be made arbitrarily small by taking the coefficients of $A^{\prime}$ sufficiently near those of $A$ uniformly in $\boldsymbol{R}^{n}$. Then the estimates (1.1) and (1.2) imply that if $A^{\prime}$ is an operator in $E\left(A_{0}, R\right)$ sufficiently near $A$ in the sense that the constant $c$ is sufficiently small in the estimate (1.3), then the dimension of $N\left(A^{\prime}\right)$ is no greater than the dimension of $N(A)$. It is easily seen via Fourier transforms that $N\left(A_{0}\right)=\{0\}$, and so, in particular, if the coefficients of an operator $A$ in $E\left(A_{0}, R\right)$ differ sufficiently little from those of $A_{0}$, then $N(A)=\{0\}$.

Given an operator $A$ in $E\left(A_{0}, R\right)$, consider the set

$$
A\left(M\left(A_{0}, R\right)\right)=\left\{A u \in L_{2}\left(R^{n} ; C^{k}\right): u \in M\left(A_{0}, R\right)\right\} .
$$

For a subset $D$ of $\boldsymbol{R}^{n}$, let $L_{2}\left(D ; C^{k}\right)$ denote the subspace of $L_{2}\left(\boldsymbol{R}^{n} ; C^{k}\right)$ consisting of those elements of $L_{2}\left(R^{n} ; C^{k}\right)$ which have support in $D$. Then not only is $A\left(M\left(A_{0}, R\right)\right)$ contained in $L_{2}\left(B_{R}^{n} ; C^{k}\right)$, but it is also an immediate consequence of the estimate (1.2) and the closedness of $A$ that $A\left(M\left(A_{0}, R\right)\right)$ is a closed subspace of $L_{2}\left(B_{R}^{n} ; C^{k}\right)$. It is shown in the sequel that the orthogonal complement of $A\left(M\left(A_{0}, R\right)\right)$ in $L_{2}\left(B_{R}^{n} ; C^{k}\right)$ is finite dimensional, from which it follows that the operator $A$ restricted to $M\left(A_{0}, R\right)$ is a bounded Fredholm operator from $M\left(A_{0}, R\right)$ to $L_{2}\left(B_{R}^{n} ; C^{k}\right)$. It is then reasonable to define the index of $A$ to be the index of this restricted Fredholm operator, for this index is invariant under small perturbations of $A$ and the range of $A$ can be characterized in terms of the range of $A_{0}$ and a finite index-related number of orthogonality conditions. If the number of independent variables is at least three, these results are more easily arrived at and more elegantly stated than in the case of two independent variables. When the number of independent variables is at least three, the index of an operator $A$ in $E\left(A_{0}, R\right)$ is, remarkably, the dimension of $N(A)$ minus the dimension of $N\left(A^{*}\right)$. This is not the case when there are only two independent variables; in fact, it is seen that the index of $A_{0}$ is $(-k)$. In the case of two independent variables, no attempt is made to express the index of an operator in $E\left(A_{0}, R\right)$ in terms of known quantities; 
rather, bounds are established for the index. The case of at least three independent variables will be dealt with first in $\S 2$; the case of two independent variables will be treated in $\S 3$.

2. The case of at least three independent variables. Suppose an unperturbed elliptic operator

$$
A_{0} u(x)=\sum_{i=1}^{n} A_{i} \frac{\partial}{\partial x_{i}} u(x)
$$

and a positive number $R$ are given, and suppose the number $n$ of independent variables is at least three. Before investigating the properties of operators in $E\left(A_{0}, R\right)$, a few preliminary lemmas are in order. Adopt the following notation: For an element $u$ of $H_{1}\left(\boldsymbol{R}^{n} ; C^{k}\right)$, put

$$
\left|\partial_{x} u(x)\right|^{2}=\sum_{i=1}^{n}\left|\frac{\partial}{\partial x_{i}} u(x)\right|^{2} \text { and }\left\|\partial_{x} u\right\|^{2}=\sum_{i=1}^{n}\left\|\frac{\partial}{\partial x_{i}} u\right\|^{2} .
$$

For $\xi$ in $\boldsymbol{R}^{n}$, put

$$
A_{0}(\xi)=\sum_{i=1}^{n} A_{i} \xi_{i}
$$

LEMMA 2.1. If $n \geqq 3$, then

$$
\int_{|x| \leqq R}|u(x)|^{2} d x \leqq \frac{R^{2}}{2(n-2)}\left\|\partial_{x} u\right\|^{2}
$$

for all $u$ in $H_{1}\left(\boldsymbol{R}^{n} ; \boldsymbol{C}^{k}\right)$.

A proof of Lemma 2.1 may be found in [6].

LEMMA 2.2. There exist positive constants $c_{1}$ and $c_{2}$ for which the estimate $c_{1}\left\|\partial_{x} u\right\| \leqq\left\|A_{0} u\right\| \leqq c_{2}\left\|\partial_{x} u\right\|$ holds for all $u$ in $H_{1}\left(\boldsymbol{R}^{n} ; C^{k}\right)$.

After taking Fourier transforms, the conclusion of Lemma 2.2 follows immediately from the ellipticity of $A_{0}$ and Parseval's Relation. Details of the proof are left to the reader.

LEMMA 2.3. If $n \geqq 3$, then $L_{2}\left(B_{R}^{n} ; C^{k}\right)$ is contained in the range of $A_{0}$.

Proof. Let $v$ be an element of $L_{2}\left(B_{R}^{n} ; C^{k}\right)$, and suppose that $M \geqq 3$. The Fourier transform $\hat{v}$ of $v$ is bounded, for

$$
|\hat{v}(\xi)| \leqq(2 \pi)^{-n / 2} \int_{|x| \leqq R}|v(x)| d x \leqq(2 \pi)^{-n / 2}\left\{\int_{|x| \leqq R} d x\right\}^{1 / 2}\|v\|,
$$

and so the function $\left|A_{0}(\xi)^{-1} \hat{v}(\xi)\right|^{2}$ is dominated by a constant multiple of $|\xi|^{-2}$ near the origin in $\boldsymbol{R}^{n}$. Since $n \geqq 3$, it follows that $-i A_{0}(\xi)^{-1} \hat{v}(\xi)$ is in $L_{2}\left(\boldsymbol{R}^{n} ; \boldsymbol{C}^{k}\right)$. The inverse Fourier transform $u$ of the function $\hat{u}(\xi)=-i A_{0}(\xi)^{-1} \hat{v}(\xi)$ is in $H_{1}\left(R^{n} ; C^{k}\right)$ and satisfies the equation $A_{0} u=v$, and the lemma is proved. 
It should be remarked that, since the $R$ appearing in the statement of Lemma 2.3 is an arbitrary positive number, it must be the case that any function in $L_{2}\left(\boldsymbol{R}^{n} ; \boldsymbol{C}^{k}\right)$ with compact support is in the range of $A_{0}$. Consider the sets

$$
L\left(A_{0}, R\right)=\left\{u \in H_{1}\left(R^{n} ; C^{k}\right): \text { support } A_{0} u \subseteq R^{n}-B_{R}^{n}\right\}
$$

and

$$
A_{0}\left(L\left(A_{0}, R\right)\right)=\left\{A_{0} u \in L_{2}\left(\boldsymbol{R}^{n} ; \boldsymbol{C}^{k}\right): u \in L\left(A_{0}, R\right)\right\}
$$

It follows from the fact that functions having compact support are dense in $L_{2}\left(R^{n}-B_{R}^{n} ; C^{k}\right)$ and from the above remark that $A_{0}\left(L\left(A_{0}, R\right)\right)$ is a dense subset of $L_{2}\left(\boldsymbol{R}^{n}-B_{R}^{n} ; \boldsymbol{C}^{k}\right)$. Furthermore, one has the following lemma.

LEMMA 2.4. If $n \geqq 3$, then every element $u$ of $H_{1}\left(R^{n} ; C^{k}\right)$ may be uniquely written as a sum $u=u_{1}+u_{2}$, where $u_{1}$ is in $L\left(A_{0}, R\right)$ and $u_{2}$ is in $M\left(A_{0}, R\right)$.

Proof. Let $u$ be an arbitrary element of $H_{1}\left(R^{n} ; C^{k}\right)$, and suppose that $n \geqq 3$. Let $X_{R}$ denote the characteristic function of $B_{R}^{n}$, i.e.

$$
\begin{aligned}
X_{R}(|x|)=1 & \text { if }|x| \leqq R \\
=0 & \text { if }|x|>R .
\end{aligned}
$$

It follows from Lemma 2.3 that $X_{R} A_{0} u$ and, hence, $A_{0} u-X_{R} A_{0} u=\left(1-X_{R}\right) A_{0} u$ are in the range of $A_{0}$. Noting that $A_{0}^{-1}$ is a well-defined linear operator from the range of $A_{0}$ to $H_{1}\left(R^{n} ; C^{k}\right)$, put $u_{1}=A_{0}^{-1}\left(1-X_{R}\right) A_{0}$ and $u_{2}=A_{0}^{-1} X_{R} A_{0} u$. Since $A_{0}\left(u-u_{1}-u_{2}\right)=0$ it must be the case that $u=u_{1}+u_{2}$. It is easily seen that this representation is unique and the proof is complete.

With these lemmas established, the investigation of properties of operators in $E\left(A_{0}, R\right)$ can be begun. Recall from the preliminary remarks that the adjoint operator $A^{*}$ of an operator $A$ in $E\left(A_{0}, R\right)$ is in $E\left(A_{0}^{*}, R\right)$. The following lemma is fundamental to subsequent investigations.

LeMma 2.5. Let $A$ be an operator in $E\left(A_{0}, R\right)$ and suppose $n \geqq 3$. Then an element of $L_{2}\left(B_{R}^{n} ; C^{k}\right)$ is orthogonal to $A\left(M\left(A_{0}, R\right)\right)$ if and only if it can be uniquely extended to an element in $L_{2}\left(\boldsymbol{R}^{n} ; \boldsymbol{C}^{k}\right)$ which is in $N\left(A^{*}\right)$.

Proof. The sufficiency is clear. To establish the necessity, let $v$ be an element of $L_{2}\left(B_{R}^{n} ; C^{k}\right)$ such that $(A u, v)=0$ for all $u$ in $M\left(A_{0}, R\right)$. It will be shown that there is a unique element $w$ of $L_{2}\left(R^{n}-B_{R}^{n} ; C^{k}\right)$ such that $(v+w)$ is in $N\left(A^{*}\right)$, i.e., such that $(A u, v+w)=0$ for all $u$ in $H_{1}\left(\boldsymbol{R}^{n} ; C^{k}\right)$.

For an arbitrary element $u$ of $H_{1}\left(\boldsymbol{R}^{n} ; C^{k}\right)$, one has

$$
\begin{aligned}
|(A u, v)| & \leqq\left\{\int_{|x| \leqq R}|A u(x)|^{2} d x\right\}^{1 / 2}\|v\| \\
& \leqq\left\{c_{1} \int_{|x| \leqq R}|u(x)|^{2} d x+c_{2} \int_{|x| \leqq R}\left|\partial_{x} u(x)\right|^{2} d x\right\}^{1 / 2}\|v\|
\end{aligned}
$$


where $c_{1}$ and $c_{2}$ are some appropriate constants depending on the norms of the coefficient matrices of $A$. Now $\int_{|x| \leqq R}\left|\partial_{x} u(x)\right|^{2} d x \leqq\left\|\partial_{x} u\right\|^{2}$ and, by Lemma 2.1,

$$
\int_{|x| \leqq R}|u(x)|^{2} d x \leqq \frac{R^{2}}{2(n-2)}\left\|\partial_{x} u\right\|^{2} .
$$

Substituting and applying Lemma 2.2 yields the estimate

$$
|(A u, v)| \leqq\left\{c_{1} R^{2} / 2(n-2)+c_{2}\right\}^{1 / 2}\left\|\partial_{x} u\right\|\|v\| \leqq c\left\|A_{0} u\right\|
$$

for some constant $c$ which does not depend on $u$. It follows from this estimate that the function $f: A_{0}\left(L\left(A_{0}, R\right)\right) \rightarrow C^{1}$ defined by $f\left(A_{0}, u\right)=-(A u, v)$ is a bounded linear functional on the dense subset $A_{0}\left(L\left(A_{0}, R\right)\right)$ of $L_{2}\left(\boldsymbol{R}^{n}-B_{R}^{n} ; \boldsymbol{C}^{k}\right)$. Hence, by the Riesz Representation Theorem, there exists a unique $w$ in $L_{2}\left(\boldsymbol{R}^{n}-B_{R}^{n} ; C^{k}\right)$ for which $\left(A_{0} u, w\right)=-(A u, v)$ for all $u$ in $L\left(A_{0}, R\right)$.

Now, given an element $u$ of $H_{1}\left(\boldsymbol{R}^{n} ; \boldsymbol{C}^{k}\right)$, write $u=u_{1}+u_{2}$, where $u_{1}$ is in $L\left(A_{0}, R\right)$ and $u_{2}$ is in $M\left(A_{0}, R\right)$. Then

$$
(A u, v+w)=\left(A\left(u_{1}+u_{2}\right), v+w\right)=\left(A u_{1}, v\right)+\left(A u_{1}, w\right)+\left(A u_{2}, v\right)+\left(A u_{2}, w\right) .
$$

The first and second terms on the right-hand side cancel, since $\left(A u_{1}, v\right)=-\left(A_{0} u_{1}, w\right)$ and since the coefficients of $A$ are equal to the coefficients of $A_{0}$ in $\boldsymbol{R}^{n}-B_{R}^{n}$. The third term on the right-hand side is zero, since $u_{2}$ is in $M\left(A_{0}, R\right)$ and $v$ is orthogonal to $A\left(M\left(A_{0}, R\right)\right)$. The fourth term on the right-hand side is zero, since the support of $A u_{2}$ is contained in $B_{R}^{n}$ and the support of $w$ is contained in $\boldsymbol{R}^{n}-B_{R}^{n}$. Consequently, $(A u, v+w)=0$ for all $u$ in $H_{1}\left(\boldsymbol{R}^{n} ; \boldsymbol{C}^{k}\right)$; in other words, $(v+w)$ is the unique extension of $v$ to an element in $N\left(A^{*}\right)$. This completes the proof.

The following theorem is an immediate consequence of Lemma 2.5.

TheOREM 2.1. Let $A$ be an operator in $E\left(A_{0}, R\right)$ and suppose $n \geqq 3$. Then the restriction of $A$ to the closed subspace $M\left(A_{0}, R\right)$ of $H_{1}\left(R^{n} ; C^{k}\right)$ is a bounded Fredholm operator from $M\left(A_{0}, R\right)$ to $L_{2}\left(B_{R}^{n} ; C^{k}\right)$, the index of which is the dimension of $N(A)$ minus the dimension of $N\left(A^{*}\right)$.

In accordance with the above theorem, define the index of an operator $A$ in $E\left(A_{0}, R\right)$ to be the dimension of $N(A)$ minus the dimension of $N\left(A^{*}\right)$; in short, Ind $A=\operatorname{dim} N(A)-\operatorname{dim} N\left(A^{*}\right)$ for an operator $A$ in $E\left(A_{0}, R\right)$. This definition is completely analogous to the usual definition of the index of a Fredholm operator. Indeed, the following theorem verifies that the index of an operator in $E\left(A_{0}, R\right)$ has the usual properties of the index of a Fredholm operator.

THeOREM 2.2. Let $A$ be an operator in $E\left(A_{0}, R\right)$ and suppose $n \geqq 3$. Then Ind $A$ $=-$ Ind $A^{*}$. Furthermore, if $A^{\prime}$ is a second operator in $E\left(A_{0}, R\right)$ which is sufficiently near $A$ in the sense that the constant $c$ is sufficiently small in the estimate (1.3), then

(i) $\operatorname{dim} N\left(A^{\prime}\right) \leqq \operatorname{dim} N(A)$ and $\operatorname{dim} N\left(A^{\prime *}\right) \leqq \operatorname{dim} N\left(A^{*}\right)$,

(ii) Ind $A^{\prime}=$ Ind $A$. 
Proof. Trivially, Ind $A=-$ Ind $A^{*}$. To establish the remainder of the theorem, note that if $A^{\prime}$ is a second operator in $E\left(A_{0}, R\right)$, then the constant $c$ in the estimate (1.3) is a bound on the norm of the restricted operator $\left(A-A^{\prime}\right)$ from $M\left(A_{0}, R\right)$ to $L_{2}\left(B_{R}^{n} ; C^{k}\right)$. The statements (i) and (ii) then follow from the basic stability theorems for Fredholm operators. (For these and other fundamental results concerning Fredholm operators, see [3] or [4].)

The lemma below is a weak version of Lemma 2.5 which is convenient for the theorem that follows.

LEMMA $2.5^{\prime}$. Let $A$ be an operator in $E\left(A_{0}, R\right)$ and suppose $n \geqq 3$. Then an element of $L_{2}\left(B_{R}^{n} ; C^{k}\right)$ is in $A\left(M\left(A_{0}, R\right)\right)$ if and only if it is orthogonal to $N\left(A^{*}\right)$.

TheOREM 2.3. Let $A$ be an operator in $E\left(A_{0}, R\right)$ and suppose $n \geqq 3$. Then an element $v$ of $L_{2}\left(\boldsymbol{R}^{n} ; \boldsymbol{C}^{k}\right)$ is in the range of $A$ if and only if $\left(1-X_{R}\right) v$ is in the range of $A_{0}$ and $\left\{X_{R} v-X_{R} A A_{0}^{-1}\left[\left(1-X_{R}\right) v\right]\right\}$ is orthogonal to $N\left(A^{*}\right)$.

Proof. To show the necessity, let $v$ be an element of $L_{2}\left(\boldsymbol{R}^{n} ; \boldsymbol{C}^{k}\right)$ which is in the range of $A$, i.e. suppose there exists an element $u$ of $H_{1}\left(R^{n} ; C^{k}\right)$ for which $A u=v$. Now

$$
A_{0} u=A u+\left(A_{0}-A\right) u=X_{R} v+\left(1-X_{R}\right) v+\left(A_{0}-A\right) u .
$$

Since $X_{R} v$ and $\left(A_{0}-A\right) u$ have support in $B_{R}^{n}$, it follows from Lemma 2.3 that there exists an element $w$ of $H_{1}\left(R^{n} ; C^{k}\right)$ for which $A_{0} w=X_{R} v+\left(A_{0}-A\right) u$. Then $A_{0}(u-w)$ $=\left(1-X_{R}\right) v$, and so $\left(1-X_{R}\right) v$ is in the range of $A_{0}$. Furthermore,

$$
\begin{aligned}
A(w) & =A(u-u+w)=v-A A_{0}^{-1}\left[\left(1-X_{R}\right) v\right] \\
& =X_{R} v-X_{R} A A_{0}^{-1}\left[\left(1-X_{R}\right) v\right]+\left(1-X_{R}\right) v-\left(1-X_{R}\right) A A_{0}^{-1}\left[\left(1-X_{R}\right) v\right] \\
& =X_{R} v-X_{R} A A_{0}^{-1}\left[\left(1-X_{R}\right) v\right]
\end{aligned}
$$

and it follows from Lemma $2.5^{\prime}$ that $\left\{X_{R} v-X_{R} A A_{0}^{-1}\left[\left(1-X_{R}\right) v\right]\right\}$ is orthogonal to $N\left(A^{*}\right)$.

To show the sufficiency, suppose that $v$ is an element of $L_{2}\left(\boldsymbol{R}^{n} ; \boldsymbol{C}^{k}\right)$ such that $\left(1-X_{R}\right) v$ is in the range of $A_{0}$ and $\left\{X_{R} v-X_{R} A A_{0}^{-1}\left[\left(1-X_{R}\right) v\right]\right\}$ is orthogonal to $N\left(A^{*}\right)$. By Lemma 2.5', there exists an element $u$ of $M\left(A_{0}, R\right)$ such that $A u=X_{R} v$ $-X_{R} A A_{0}^{-1}\left[\left(1-X_{R}\right) v\right]$. Then

$$
\begin{aligned}
A\left(u+A_{0}^{-1}\left[\left(1-X_{R}\right) v\right]\right)= & X_{R} v-X_{R} A A_{0}^{-1}\left[\left(1-X_{R}\right) v\right]+A A_{0}^{-1}\left[\left(1-X_{R}\right) v\right] \\
= & X_{R} v-X_{R} A A_{0}^{-1}\left[\left(1-X_{R}\right) v\right]+X_{R} A A_{0}^{-1}\left[\left(1-X_{R}\right) v\right] \\
& +\left(1-X_{R}\right) A A_{0}^{-1}\left[\left(1-X_{R}\right) v\right] \\
= & X_{R} v+\left(1-X_{R}\right) v=v .
\end{aligned}
$$

Thus $v$ is in the range of $A$, and the theorem is proved.

The above theorem provides the promised characterization of the range of an operator in $E\left(A_{0}, R\right)$ in terms of the range of $A_{0}$ and a finite number of orthogonality conditions. The following is an interesting corollary: If $A$ is an operator 
in $E\left(A_{0}, R\right)$ for which $N\left(A^{*}\right)=\{0\}$ and if $n \geqq 3$, then the range of $A$ is precisely the range of $A_{0}$. This completes these investigations for the case of at least three independent variables.

3. The case of two independent variables. Suppose there are given a positive $R$ and an unperturbed elliptic operator

$$
A_{0} u(x)=\sum_{i=1}^{2} A_{i} \frac{\partial}{\partial x_{i}} u(x)
$$

acting on elements $u$ of $H_{1}\left(R^{2} ; C^{k}\right)$. Since the number of independent variables is two, the situation at hand differs considerably from the case treated in the previous section. For one thing, there is no analogue of the estimate of Lemma 2.1, as may be seen by considering the following sequence of functions in $H_{1}\left(R^{2} ; C^{k}\right)$ defined for $m=2,3, \ldots$ :

$$
\begin{aligned}
u_{m}(x) & =1 & & \text { if }|x| \leqq 1, \\
& =\frac{-1}{\log m} \int_{m}^{|x|} \frac{1}{t} d t & & \text { if } 1<|x| \leqq m, \\
& =0 & & \text { if }|x|>m .
\end{aligned}
$$

For these functions, one has $\int_{|x| \leqq 1}\left|u_{m}(x)\right|^{2} d x=\pi$ while

$$
\int_{R^{2}}\left|\partial_{x} u_{m}(x)\right|^{2} d x=\frac{2 \pi}{\log m}
$$

For another thing, not all of $L_{2}\left(B_{R}^{2} ; C^{k}\right)$ is contained in the range of $A_{0}$; hence, modified forms of Lemma 2.3 and Lemma 2.4 must be presented. Lemma 2.2, however, remains valid, i.e., there exist positive constants $c_{1}$ and $c_{2}$ for which the estimate

$$
c_{1}\left\|\partial_{x} u\right\| \leqq\left\|A_{0} u\right\| \leqq c_{2}\left\|\partial_{x} u\right\|
$$

holds for all $u$ in $H_{1}\left(R^{2} ; C^{k}\right)$.

The following lemma characterizes those elements of $L_{2}\left(B_{R}^{2} ; C^{k}\right)$ which lie in the range of $A_{0}$.

LEMMA 3.1. Let $v$ be an element of $L_{2}\left(B_{R}^{2} ; C^{k}\right)$. Then the following are equivalent:

(i) $v$ is in the range of $A_{0}$.

(ii) The Fourier transform $\hat{v}$ of $v$ vanishes at the origin.

(iii) $v$ is orthogonal in $L_{2}\left(R^{2} ; C^{k}\right)$ to all functions of the form $X_{R} c$, where $c$ is a vector in $C^{k}$.

Proof. Clearly, (ii) and (iii) are equivalent. To show the equivalence of (i) and (ii), note that $v$ is in the range of $A_{0}$ if and only if the Fourier transform $\hat{v}$ of $v$ is such that the function $-i A_{0}(\xi)^{-1} \hat{v}(\xi)$ is in $L_{2}\left(R^{2} ; C^{k}\right)$. Since $v$ has compact support, $\hat{v}(\xi)$ is a continuously differentiable function of $\xi$. It follows that $-i A_{0}(\xi)^{-1} \hat{v}(\xi)$ is in $L_{2}\left(\boldsymbol{R}^{2} ; \boldsymbol{C}^{k}\right)$ if and only if $\hat{v}(0)=0$, and the proof is complete. 
The definition of the set $L\left(A_{0}, R\right)$ must be altered to serve the present circumstances. Redefine

$$
L\left(A_{0}, R\right)=\left\{u \in H_{1}\left(\boldsymbol{R}^{2} ; C^{k}\right): A_{0} u \text { is constant in } B_{R}^{2}\right\} .
$$

The following lemma is the desired modification of Lemma 2.4.

LEMMA 3.2. Every element $u$ of $H_{1}\left(\boldsymbol{R}^{2} ; C^{k}\right)$ may be uniquely written as a sum $u=u_{1}+u_{2}$ where $u_{1}$ is in $L\left(A_{0}, R\right)$ and $u_{2}$ is in $M\left(A_{0}, R\right)$.

Proof. Let $u$ be an element of $H_{1}\left(\boldsymbol{R}^{2} ; C^{k}\right)$. Then the function

$$
X_{R}(|x|) A_{0} u(x)-X_{R}(|x|)\left(2 / R^{2}\right)\left[\left(X_{R} A_{0} u\right)^{\wedge}(0)\right]
$$

is in $L_{2}\left(B_{R}^{2} ; C^{k}\right)$, and its Fourier transform vanishes at the origin. By Lemma 3.1, there exists an element $u_{2}$ of $M\left(A_{0}, R\right)$ such that

$$
A_{0} u_{2}=X_{R} A_{0} u-X_{R}\left(2 / R^{2}\right)\left[\left(X_{R} A_{0} u\right)^{\wedge}(0)\right] .
$$

The function $u_{1}=u-u_{2}$ satisfies

$$
\begin{aligned}
A_{0} u_{1} & =A_{0} u-X_{R} A_{0} u+X_{R}\left(2 / R^{2}\right)\left[\left(X_{R} A_{0} u\right)^{\wedge}(0)\right] \\
& =\left(1-X_{R}\right) A_{0} u+X_{R}\left(2 / R^{2}\right)\left[\left(X_{R} A_{0} u\right)^{\wedge}(0)\right],
\end{aligned}
$$

and it is immediately seen that $u_{1}$ is in $L\left(A_{0}, R\right)$ and that $u=u_{1}+u_{2}$ as desired. The uniqueness of this representation follows from the fact that $N\left(A_{0}\right)=\{0\}$ and from the fact that the sets $A_{0}\left(L\left(A_{0}, R\right)\right)$ and $A_{0}\left(M\left(A_{0}, R\right)\right)$ are orthogonal in $L_{2}\left(\boldsymbol{R}^{2} ; C^{k}\right)$. This proves the lemma.

To begin the investigation of the properties of operators in $E\left(A_{0}, R\right)$, consider the following lemma.

LEMMA 3.3. If $A$ is an operator in $E\left(A_{0}, R\right)$ which has no zero-order term, then the dimension of the orthogonal complement of $A\left(M\left(A_{0}, R\right)\right)$ in $L_{2}\left(B_{R}^{2} ; C^{k}\right)$ is at least $\operatorname{dim} N\left(A^{*}\right)$ and at most $\operatorname{dim} N\left(A^{*}\right)+k$.

Proof. First note that the restriction of any element of $N\left(A^{*}\right)$ to $B_{R}^{2}$ is orthogonal in $L_{2}\left(B_{R}^{2} ; C^{k}\right)$ to $A\left(M\left(A_{0}, R\right)\right)$. Hence, the dimension of the orthogonal complement of $A\left(M\left(A_{0}, R\right)\right)$ in $L_{2}\left(B_{R}^{2} ; C^{k}\right)$ is at least $\operatorname{dim} N\left(A^{*}\right)$. Now let arbitrary elements $v_{1}, \ldots, v_{k+1}$ in the orthogonal complement of $A\left(M\left(A_{0}, R\right)\right)$ in $L_{2}\left(B_{R}^{2} ; C^{k}\right)$ be given. The lemma will be proved if it can be shown that there exist scalars $\alpha_{1}, \ldots, \alpha_{k+1}$, not all zero, such that the linear combination $\alpha_{1} v_{1}+\cdots+\alpha_{k+1} v_{k+1}$ can be extended to an element of $N\left(A^{*}\right)$.

Suppose that $v$ is any element of $L_{2}\left(B_{R}^{2} ; C^{k}\right)$. Since $A$ has bounded coefficients and no zero-order term, there exists a positive constant $c$ for which the estimate $|(A u, v)| \leqq\|A u\|\|v\| \leqq c\left\|\partial_{x} u\right\|$ holds for all $u$ in $H_{1}\left(\boldsymbol{R}^{2} ; C^{k}\right)$. It follows from Lemma 2.2 that there is a positive constant $C$ for which the estimate $|(A u, v)| \leqq C\left\|A_{0} u\right\|$ holds for all $u$ in $H_{1}\left(R^{2} ; C^{k}\right)$. This implies, in particular, that the function $f: A_{0}\left(L\left(A_{0}, R\right)\right) \rightarrow C^{1}$ defined by $f\left(A_{0} u\right)=-(A u, v)$ is a bounded linear functional 
on the linear submanifold $A_{0}\left(L\left(A_{0}, R\right)\right)$ of $L_{2}\left(\boldsymbol{R}^{2} ; \boldsymbol{C}^{k}\right)$. Then it follows from the Riesz Representation Theorem that there exists a unique $w$ in the $L_{2}\left(\boldsymbol{R}^{2} ; \boldsymbol{C}^{k}\right)$ closure of $A_{0}\left(L\left(A_{0}, R\right)\right)$ for which $\left(A_{0} u, w\right)=-(A u, v)$ for all $u$ in $L\left(A_{0}, R\right)$. Note that $w$ is constant inside the ball $B_{R}^{2}$.

It follows from this discussion that there exist elements $w_{1}, \ldots, w_{k+1}$ in the $L_{2}\left(\boldsymbol{R}^{2} ; C^{k}\right)$-closure of $A_{0}\left(L\left(A_{0}, R\right)\right)$ which are constant inside $B_{R}^{2}$ and which satisfy $\left(A_{0} u, w_{i}\right)=-\left(A u, v_{i}\right), i=1, \ldots, k+1$, for all $u$ in $L\left(A_{0}, R\right)$. Let $\alpha_{1}, \ldots, \alpha_{k+1}$ be some collection of scalars, not all zero, such that the linear combination $w_{0}$ $=\alpha_{1} w_{1}+\cdots+\alpha_{k+1} w_{k+1}$ vanishes inside the ball $B_{R}^{2}$. Suppose that $u$ is an arbitrary element of $H_{1}\left(\boldsymbol{R}^{2} ; \boldsymbol{C}^{k}\right)$. Writing $u=u_{1}+u_{2}$, where $u_{1}$ and $u_{2}$ belong to $L\left(A_{0}, R\right)$ and $M\left(A_{0}, R\right)$, respectively, one has, as in the proof of Lemma 2.5,

$$
\begin{aligned}
\left(A u, \sum_{i=1}^{k+1} \alpha_{i} v_{i}+w_{0}\right) & =\left(A u_{1}, \sum_{i=1}^{k+1} \alpha_{i} v_{i}\right)+\left(A u_{1}, w_{0}\right)+\left(A u_{2}, \sum_{i=1}^{k+1} \alpha_{i} v_{i}\right)+\left(A u_{2}, w_{0}\right) \\
& =-\left(A u_{1}, w_{0}\right)+\left(A_{0} u_{1}, w_{0}\right)+0+0=0
\end{aligned}
$$

Consequently, the element $w_{0}$ of $L_{2}\left(\boldsymbol{R}^{2}-B_{R}^{2} ; \boldsymbol{C}^{k}\right)$ extends the linear combination $\sum_{i=1}^{k+1} \alpha_{i} v_{i}$ to an element of $N\left(A^{*}\right)$, and the lemma is proved.

To study arbitrary operators in $E\left(A_{0}, R\right)$, adopt the following notation: For an operator

$$
A u(x)=\sum_{i=1}^{2} A_{i}(x) \frac{\partial}{\partial x_{i}} u(x)+B(x) u(x)
$$

in $E\left(A_{0}, R\right)$, denote by

$$
A_{f} u(x)=\sum_{i=1}^{2} A_{i}(x) \frac{\partial}{\partial x_{i}} u(x)
$$

the operator consisting of the first-order terms of $A$.

THEOREM 3.1. Let $A$ be an operator in $E\left(A_{0}, R\right)$. Then the restriction of $A$ to the closed subspace $M\left(A_{0}, R\right)$ of $H_{1}\left(R^{2} ; C^{k}\right)$ is a bounded Fredholm operator from $M\left(A_{0}, R\right)$ to $L_{2}\left(B_{R}^{2} ; C^{k}\right)$, the index of which is at least $\left[\operatorname{dim} N\left(A_{f}\right)-\operatorname{dim} N\left(A_{f}^{*}\right)-k\right]$ and at most $\min \left\{\left[\operatorname{dim} N(A)-\operatorname{dim} N\left(A^{*}\right)\right]\right.$, $\left.\left[\operatorname{dim} N\left(A_{f}\right)-\operatorname{dim} N\left(A_{f}^{*}\right)\right]\right\}$.

Proof. It is an obvious corollary of Lemma 3.3 that the restriction of $A_{f}$ to $M\left(A_{0}, R\right)$ is a bounded Fredholm operator from $M\left(A_{0}, R\right)$ to $L_{2}\left(B_{R}^{2} ; C^{k}\right)$ whose index lies between [dim $\left.N\left(A_{f}\right)-\operatorname{dim} N\left(A_{f}^{*}\right)-k\right]$ and $\left[\operatorname{dim} N\left(A_{f}\right)-\operatorname{dim} N\left(A_{f}^{*}\right)\right]$. To extend this statement to include the operator $A$, consider the following observation: If $B(x)$ is a continuous $k \times k$ matrix-valued function on $\boldsymbol{R}^{2}$ which vanishes outside the ball $B_{R}^{2}$, then the operator

$$
B: M\left(A_{0}, R\right) \rightarrow L_{2}\left(B_{R}^{2} ; C^{k}\right)
$$

defined by $B u(x)=B(x) u(x)$ is a compact operator. (This follows immediately from the Rellich Compactness Theorem [1, p. 169] if $B(x)$ is a continuously differ- 
entiable function of $x$. If $B(x)$ is merely continuous in $x$, then it may be approximated arbitrarily closely uniformly on $\boldsymbol{R}^{n}$ by $k \times k$ matrix-valued functions which are differentiable functions of $x$. This implies that the operator $B$ defined by $B(x)$ can be approximated arbitrarily closely in norm by compact operators and, hence, must be compact.) From this observation, it is seen that the restrictions of $A$ and $A_{f}$ to $M\left(A_{0}, R\right)$ differ by a compact operator. It is then an immediate consequence of the stability properties of Fredholm operators with respect to compact perturbations that the restriction of $A$ to $M\left(A_{0}, R\right)$ is a Fredholm operator from $M\left(A_{0}, R\right)$ to $L_{2}\left(B_{R}^{2} ; C^{k}\right)$ whose index is equal to the index of the restriction of $A_{f}$ to $M\left(A_{0}, R\right)$. Since, clearly, the index of the restriction of $A$ to $M\left(A_{0}, R\right)$ is no greater than [dim $N(A)-\operatorname{dim} N\left(A^{*}\right)$ ], it follows that the index of the restriction of $A$ to $M\left(A_{0}, R\right)$ is at least $\left[\operatorname{dim} N\left(A_{f}\right)-\operatorname{dim} N\left(A_{f}^{*}\right)-k\right]$ and at most

$$
\min \left\{\left[\operatorname{dim} N(A)-\operatorname{dim} N\left(A^{*}\right)\right],\left[\operatorname{dim} N\left(A_{f}\right)-\operatorname{dim} N\left(A_{f}^{*}\right)\right]\right\} .
$$

This completes the proof.

As in the preceding section, one may define the index Ind $A$ of an operator $A$ in $E\left(A_{0}, R\right)$ to be the index of the restriction of $A$ to a bounded Fredholm operator from $M\left(A_{0}, R\right)$ to $L_{2}\left(B_{R}^{2} ; C^{k}\right)$. Unfortunately, the dependence of Ind $A$ on $A$ in the present circumstance is much less elegant than in the case of at least three independent variables. Note, in particular, that it is no longer true that Ind $A$ $=-$ Ind $A^{*}$. (In fact, it follows from Lemma 3.1 that Ind $A_{0}=\operatorname{Ind} A_{0}^{*}=-k$.) Consequently, no analogue of Theorem 2.2 will be presented. Let it suffice to say that, as before, the index of an operator $A$ in $E\left(A_{0}, R\right)$ is invariant under small perturbations of $A$ in $E\left(A_{0}, R\right)$. The following theorem is the best possible resurrection of Theorem 2.3; it concludes these investigations for the case of two independent variables.

THEOREM 3.2. Let $A$ be an operator in $E\left(A_{0}, R\right)$. Then an element $v$ of $L_{2}\left(\boldsymbol{R}^{2} ; \boldsymbol{C}^{k}\right)$ is in the range of $A$ if and only if there exists a vector $c$ in $C^{k}$ such that $\left[\left(1-X_{R}\right) v+X_{R} c\right]$ is in the range of $A_{0}$ and $\left\{X_{R} v-X_{R} A A_{0}^{-1}\left[\left(1-X_{R}\right) v+X_{R} c\right]\right\}$ is in $A\left(M\left(A_{0}, R\right)\right)$, a closed subspace of $L_{2}\left(B_{R}^{2} ; C^{k}\right)$ whose orthogonal complement in $L_{2}\left(B_{R}^{2} ; C^{k}\right)$ has dimension $[\operatorname{dim} N(A)-$ Ind $A]$.

Proof. To show the necessity, let $v$ be an element of $L_{2}\left(\boldsymbol{R}^{2} ; \boldsymbol{C}^{k}\right)$ which is in the range of $A$, i.e. let there exist an element $u$ of $H_{1}\left(R^{2} ; C^{k}\right)$ for which $A u=v$. Now

$$
A_{0} u=A u+\left(A_{0}-A\right) u=\left(1-X_{R}\right) v+X_{R} v+\left(A_{0}-A\right) u .
$$

Since $\left[X_{R} v+\left(A_{0}-A\right) u\right]$ has support in $B_{R}^{2}$, it follows from Lemma 3.1 that there exists a vector $c$ in $C^{k}$ and an element $w$ of $H_{1}\left(\boldsymbol{R}^{2} ; \boldsymbol{C}^{k}\right)$ for which $A_{0} w=X_{R} v$ $+\left(A_{0}-A\right) u-X_{R} c$. Then

$$
A_{0}(u-w)=\left(1-X_{R}\right) v+X_{R} c
$$


and so $\left[\left(1-X_{R}\right) v+X_{R} c\right]$ is in the range of $A_{0}$. Furthermore,

$$
\begin{aligned}
A(w) & =A(u-u+w)=v-A A_{0}^{-1}\left[\left(1-X_{R}\right) v+X_{R} c\right] \\
& =X_{R} v-X_{R} A A_{0}^{-1}\left[\left(1-X_{R}\right) v+X_{R} c\right],
\end{aligned}
$$

and it follows that $\left\{X_{R} v-X_{R} A A_{0}^{-1}\left[\left(1-X_{R}\right) v+X_{R} c\right]\right\}$ is in $A\left(M\left(A_{0}, R\right)\right)$.

To show the sufficiency, suppose that $v$ is an element of $L_{2}\left(\boldsymbol{R}^{2} ; C^{k}\right)$ and that $c$ is a vector in $C^{k}$ such that $\left[\left(1-X_{R}\right) v+X_{R} c\right]$ is in the range of $A_{0}$ and $A u=X_{R} v$ $-X_{R} A A_{0}^{-1}\left[\left(1-X_{R}\right) v+X_{R} c\right]$ for some $u$ in $M\left(A_{0}, R\right)$. Then

$$
\begin{aligned}
A\left(u+A_{0}^{-1}\right. & {\left.\left[\left(1-X_{R}\right) v+X_{R} c\right]\right) } \\
= & X_{R} v-X_{R} A A_{0}^{-1}\left[\left(1-X_{R}\right) v+X_{R} c\right]+X_{R} A A_{0}^{-1}\left[\left(1-X_{R}\right) v+X_{R} c\right] \\
& +\left(1-X_{R}\right) A A_{0}^{-1}\left[\left(1-X_{R}\right) v+X_{R} c\right] \\
= & X_{R} v+\left(1-X_{R}\right) v=v .
\end{aligned}
$$

Thus $v$ is in the range of $A$ and the theorem is proved.

\section{REFERENCES}

1. L. Bers, F. John and M. Schecter, Partial differential equations, Proc. Summer Seminar (Boulder, Colorado, 1957), Lectures in Appl. Math., vol. 3, Interscience, New York, 1964. MR 29 \#346.

2. K. O. Friedrichs, The identity of weak and strong extensions of differential operators, Trans. Amer. Math. Soc. 55 (1944), 132-151. MR 5, 188.

3. I. C. Gohberg and M. G. Krein, The basic propositions on defect numbers, root numbers and indices of linear operators, Uspehi Mat. Nauk 12 (1957), no. 2 (74), 43-118; English transl., Amer. Math. Soc. Transl. (2) 13 (1960), 185-264. MR 20 \#3459; MR 22 \#3984.

4. T. Kato, Perturbation theory for linear operators, Die Grundlehren der math. Wissenschaften, Band 132, Springer-Verlag, New York, 1966. MR 34 \#3324.

5. P. D. Lax and R. S. Phillips, Lectures on scattering theory, Summer Institute on Scattering Theory, Northern Arizona University, Flagstaff, Arizona, 1969.

6. ——, Scattering theory, Pure and Appl. Math., vol. 26, Academic Press, New York, 1967. MR $36 \# 530$.

7. H. F. Walker, On the null-spaces of first-order elliptic partial differential operators in $R^{n}$, Proc. Amer. Math. Soc. (to appear).

Department of Mathematics, Texas Tech University, Lubbock, Texas 79409 